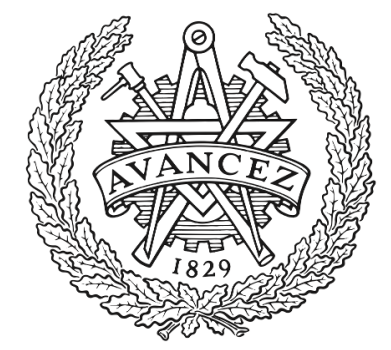

\title{
CHALMERS
}

UNIVERSITY OF TECHNOLOGY

\section{Transmitter comparison and unequal bit error probabilities in coherent QPSK systems}

Downloaded from: https://research.chalmers.se, 2023-04-26 14:53 UTC

Citation for the original published paper (version of record):

Zhao, H., Karlsson, M., Agrell, E. (2007). Transmitter comparison and unequal bit error probabilities in coherent QPSK systems. Optical Fiber Communication Conference and the National Fiber Optic Engineers Conference: OTuH2-. http://dx.doi.org/10.1109/OFC.2007.4348798

N.B. When citing this work, cite the original published paper. 


\title{
Transmitter Comparison and Unequal Bit Error Probabilities in Coherent QPSK Systems
}

\author{
Hongxia Zhao*, Magnus Karlsson**, Erik Agrell* \\ * Communications Systems Group, Department of Signals and Systems \\ ** Photonics Laboratory, Department of Microtechnology and Nanoscience \\ Chalmers University of Technology, \\ SE-412 96 Gothenburg, Sweden \\ email:hz@chalmers.se,magnus.karlsson@mc2.chalmers.se, agrell@s2.chalmers.se
}

\begin{abstract}
We compare different QPSK transmitters and find that some simple configurations can give rise to a significant difference in BER between the two transmitted bits. The optimum receiver filter bandwidth is affected by this phenomenon.

(C) 2006 Optical Society of America

OCIS codes: (060.2330) Fiber Optics Communications; 060.4080 Modulation
\end{abstract}

\section{Introduction}

Coherent transmission technologies are receiving renewed interest [1-4], e.g. for its increased sensitivities [1], enabling denser packing of WDM channels [2], the potential of electrical dispersion compensation $[1,3]$ and enabling sophisticated coded multilevel modulation formats [4]. In particular, quadrature phase-shift keying (QPSK) is considered as an interesting trade-off between complexity and performance, as it offers doubled transmitted data rates with maintained bandwidth and sensitivity, compared to e.g. on-off keying (OOK). QPSK has a long history of successful use in wireless transmission systems, but optical systems are unique in that the higher bit rates make bandwidth limitations from e.g. modulators, detectors, and filters non-negligible, and a cause of inter-symbol interference (ISI) [5]. In fact, most optical systems are always designed so that the electronic receiver bandwidth is optimized to yield the best trade off between noise suppression and ISI [6]. In this paper we investigate for the first time this trade-off for QPSK transmission, and compare the performance of different simple, yet realistic, transmitter configurations and different filter bandwidths. Earlier QPSK transmitter comparisons have focused on either differential modulation [7] or other transmitter aspects [8]. We report here a novel characteristic, i.e. that the two bits in the transmitted QPSK symbol have different bit-error rates (BERs), and explain this as originating from the combined ISI in the modulator and receiver filter. We also show how to suppress this phenomenon by a simple modification of the transmitter.

\section{Simulation Model}

A back-to-back coherent QPSK fiber optic system with a symbol rate of 10 Gsymbols/s is investigated. The study will focus on the transmitter and receiver properties. Thus, effects of fiber dispersion and nonlinearity will not be included in this paper. The optical amplified spontaneous emission noise is the dominant noise source; we therefore ignore the thermal and shot noise in our study.

Three QPSK transmitters have been investigated, as shown in Figure 1. Transmitter 1 (PM) has a single phase modulator, while transmitter 2 (MZM-PM) and 3 (par-MZM) have serial or parallel modulators, respectively. The driving signals to these modulators are Raised Cosine (RC) impulses as defined in [7].

The receiver consists of an optical EDFA preamplifier with gain and noise figure set to be $30 \mathrm{~dB}$ and $5 \mathrm{~dB}$, respectively, followed by a $40 \mathrm{GHz}$ optical Gaussian bandpass filter to suppress spontaneous-spontaneous beat noise. Then a local oscillator (LO) is mixed with the signal in a 90 degree hybrid before detection. We assume the LO and signal to be software synchronized [1-3], so that the LO phase noise can be negligible with respect to the optical amplifier noise. The carrier wavelength equals to $1550 \mathrm{~nm}$ and the electric signal is finally filtered with third-order Butterworth LP filters of variable bandwidth, that will be discussed below. Monte-Carlo simulations using random independent information bits with a total bit rate of $20 \mathrm{~Gb} / \mathrm{s}$ were then performed, and the BER was estimated after 50-100 bit errors had occurred. 


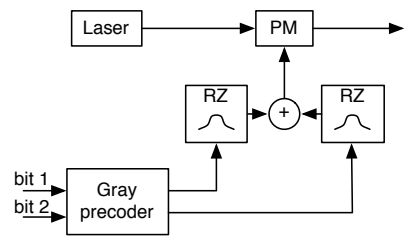

PM

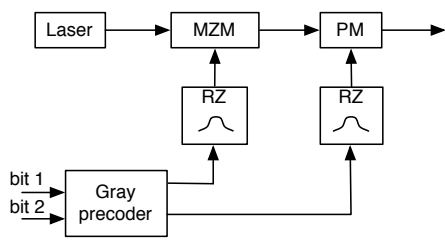

MZM-PM

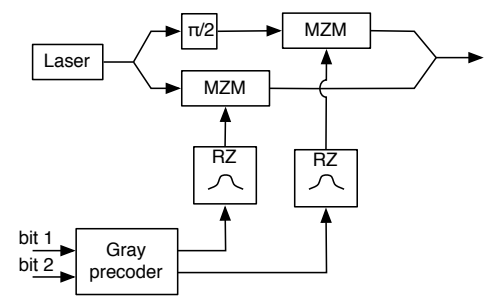

par-MZM

Fig. 1. The single phase modulator (PM), the serial MZM-PM, and the parallel MZM (par-MZM). The input bits are Gray precoded, and raised cosine (RC) shaped before driving the modulators.
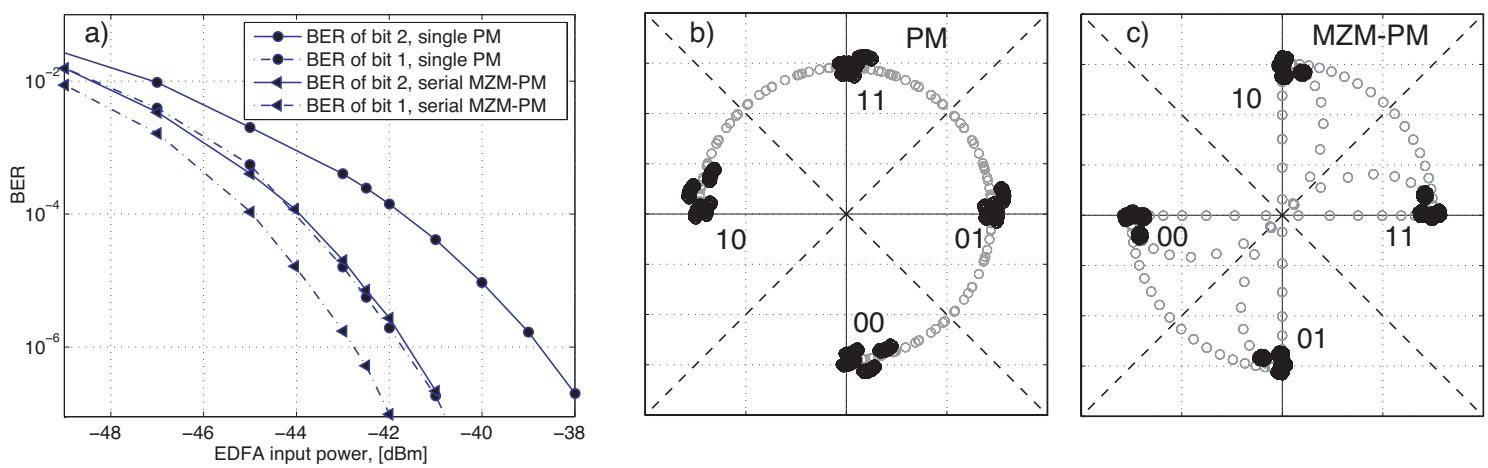

Fig. 2. a): BER curves for the respective bits of the PM and MZM-PM different transmitters. b): Constellation of signals from the single PM transmitter c): Constellations from the MZM-PM transmitter. The grey circles denote the time variation directly after transmitter, and black circles show received data samples after $7 \mathrm{GHz}$ electric filtering. The dashed lines denote decision boundaries.

\section{Results and discussion}

Typical BER curves for the respective bits for the PM and MZM-PM transmitters are shown in Fig. 2a). A significant difference in BER between the bits is found for both transmitter types, and it can be explained by the constellation diagrams shown in Fig. 2 b)-c). These constellations are plotted without any noise, and show thus only the influence from the ISI. It may be shown that the ISI is a combination of the transmitterinduced ISI (which causes movement along the gray circles in the constellation diagrams of Fig 2) and the low-pass filter ISI in the receiver (which causes splitting of each signal level in 4 separate clusters). The total ISI gives rise to an asymmetric overall movement of the signal levels towards the bit 2 decision boundary, with a concomitant increase of errors in bit 2. An order of magnitude difference in BER between the bits is seen in our example, and as the total system BER is the average of the bitwise BERs, the worst bit will dominate and significantly degrade the overall performance.

In fig 3a), solid lines, we show (for the PM transmitter) how the BER performance depends on the electrical filter bandwidth, and clearly the trade-off between ISI and SNR will differ between the two bits, since the ISI influences them differently. The optimum filter bandwidths can be between 7 and $9 \mathrm{GHz}$, and as can be seen it differs significantly between the bits. A similar behavior is found for the MZM-PM transmitter. We next verified that a simple pre-emphasis of the PM-transmitter, intended to optimize the constellation positions in presence of the ISI, significantly improved the performance, and this modified system (denoted mod-PM) is shown as dashed lines in Fig. 3a). We now see that the worst bit is improved, at a slight expense of the other bit, but yet it will lower the average BER. For the modified transmitter, both bits have almost the same optimum bandwidth around 7.5 GHz. The SNR used in Fig 3a) equals $11 \mathrm{~dB}$ (in terms of the bit energy per noise power density $E_{B} / N_{0}$ after electrical lowpass filtering) which corresponds to a received optical power of $-43 \mathrm{dBm}$.

In Fig 3b) the BER performance for a number of different transmitters are compared, together with the 

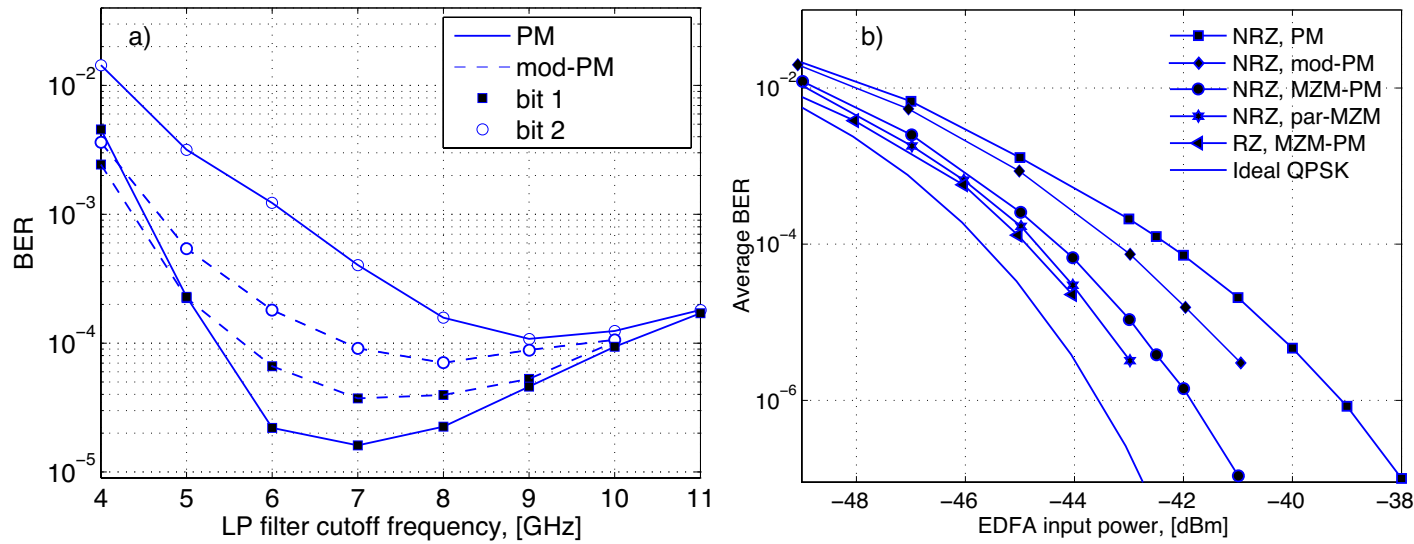

Fig. 3. a): Plot of the BER for the two bits vs electric filter bandwidth for the PM and modified PM transmitters. b): BER performance comparison of a QPSK coherent system using different transmitters. The solid line is the theoretical maximum, i.e. the Gaussian QPSK channel.

ideal Gaussian QPSK channel. This is now the total BER, i.e. the average of both bits. In all cases a filter bandwidth of $7 \mathrm{~dB}$ was used. The single-PM has the worst performance, but is on the other hand the simplest transmitter, hardware-wise. Its sensitivity can be improved with $1 \mathrm{~dB}$ at a BER of $10^{-6}$ by the simple modification outlined above (mod-PM), and we believe this improvement can be extrapolated to $2 \mathrm{~dB}$ at a BER of $10^{-9}$, although a substantial simulation effort would be needed to prove this. The MZM-PM and par-MZM transmitters have closer performance to an RZ-modulated MZM-PM transmitter. In the last case, an additional amplitude modulator was used for pulse carving. Note however that all improvements over the simplest PM transmitter and mod-PM transmitter are obtained at the prize of an increased hardware complexity.

In conclusion, we have compared a number of QPSK transmitter configurations, and found an unequal BER in the simplest cases (PM, MZM-PM), that limits the performance. A certain improvement is realized by pre-emphasizing the PM, and even better performance is found when using RZ amplitude modulation, similarly to DPSK [6]. However this comes at the expense of a higher bandwidth and/or increased transmitter complexity.

We would like to acknowledge P. A. Andrekson for discussions and comments, and the Swedish Scientific Council (VR) for funding.

\section{References}

1. M. Taylor, "Coherent Detection for Fiber Optic Communications Using Digital Signal Processing", paper CThB1, in topical meeting on Coherent Optical Technologies and Applications (COTA), Whistler, Canada, Jun. 2006.

2. D. Ly-Gagnon, S. Tsukamoto, K. Katoh, and K. Kikuchi, "Coherent detection of optical quadrature phase-shift keying signals with carrier phase estimation", IEEE J. Lightwave Tech. 24, pp. 1221, 2006.

3. S. Tsukamoto, K. Katoh, and K. Kikuchi, "Unrepeated Transmission of 20-Gb/s Optical Quadrature Phase-Shift-Keying Signal Over 200-km Standard Single-Mode Fiber Based on Digital Processing of Homodyne-Detected Signal for GroupVelocity Dispersion Compensation", IEEE Phot. Technol. Lett., 18, pp. 1016-1018, 2006.

4. H. Zhao, E. Agrell, and M. Karlsson, "Trellis-coded modulation in PSK and DPSK Communications", paper We3.P.93, European Conference on Optical Communications, ECOC 2006

5. N. Kikuchi, K. Sekine, and S. Sasaki, "Multilevel signalling for high-speed optical transmission", paper Tu3.2.1, and "Proposal of Intersymbol interference (ISI) suppression technique for optical multilevel signal generation", paper Tu 4.2.1, European Conference on Optical Communications, ECOC 2006

6. P. J. Winzer, S. Chandrasekhar, and H. Kim, "Impact of filtering on RZ-DPSK reception", IEEE Photon. Technol. Lett., vol. 15, no. 6, pp. 840842, Jun. 2003.

7. M. Ohm and T. Freckmann, "Comparison of different DQPSK transmitters with NRZ and RZ impulse shaping", in IEEE/LEOS Workshop on Advanced Modulation Formats, SanFrancisco, USA, Jul. 2004.

8. L. Christen and A. E. Willner, "System sensitivity of multi-level 16-QAM and QPSK to transmitter imperfections indifferent modulator designs", in topical meeting on Coherent Optical Technologies and Applications (COTA), Whistler, Canada, Jun. 2006. 